\title{
PROKLA-Redaktion
}

\section{Editorial: Gesellschaftskritik und 150 Jahre Kritik der politischen Ökonomie}

Seit einigen Jahren hat Gesellschaftskritik in der Soziologie (wieder) Hochkonjunktur - und mit ihr die disziplinäre Selbstverständigung darüber, welche Möglichkeiten und Aufgaben einer (wie auch immer zu definierenden) „kritischen“ Soziologie zukommen sollte. Der Heftschwerpunkt „Perspektiven der Gesellschaftskritik“ widmet sich der Frage, ob die neueren Debatten über sozialwissenschaftliche Kritik in der gegenwärtigen Situation veränderte Bedeutung erhalten. Ausgangspunkt hierfür ist zunächst eine doppelte Wahrnehmung: Zum einen, dass es in Teilen des akademischen Diskurses - nachdem Gesellschaftskritik der etablierten Soziologie jahrelang als anrüchige Tätigkeit galt - zu einer erfreulichen Wiederkehr der Gesellschaftskritik gekommen ist; zum anderen jedoch die entsprechenden Debatten häufig merkwürdig formal und abstrakt bleiben. Zahlreiche Monografien und Sammelbände haben die Facetten sozialwissenschaftlicher und politisch-philosophischer "Kritik“ erkundet, dabei aber häufig die "Sachkritik“, das heißt die Kritik der konkreten gesellschaftlichen Verhältnisse, zugunsten umfassender selbstreflexiver Verständigungen vernachlässigt. Oftmals wurde und wird nicht deutlich, wer oder was - und warum eigentlich - von wem kritisiert werden soll.

Angesichts dessen rekonstruiert der Beitrag von Tilman Reitz die gegenwärtige Kritik-Debatte mit Blick auf jüngere Ausprägungen der kritischen Theorie (u.a. an der Frankfurter Universität). Sein Beitrag zeigt, dass und wie die abstrakte Konzentration auf die Reflexion kritischer Prinzipien und Verfahrensweisen den Anspruch sachhaltiger und politisch wirksamer Kritik untergräbt. Er diskutiert, wie sich diese Selbstbezüglichkeit aus den akademischen Rahmenbedingungen der Kritikproduktion erklären lässt. Silke van $D y k$ widmet sich dem (derzeit vor allem mit dem „System Trump \& Co.“verbundenen) Phänomen der „alternativen Fakten“ und untersucht gegenwärtige Wahrheitsregime von rechts und die Möglichkeiten und Leerstellen ihrer emanzipatorischen, namentlich poststrukturalistisch inspirierten Kritik. Keine leichte Aufgabe, bestand doch ein wesentlicher Impuls letzterer Theorieströmungen in der prinzipiellen Dekonstruktion von Wahrheitsansprüchen. Dies macht die Aufgabe jedoch umso dringlicher, eine Kritik gegenwärtiger rechtspopulistischer (Un-)Wahrheitsspiele auszuarbeiten, die sich selbst einem Positivismus vermeintlich unbestreitbarer Fakten entzieht und zugleich die Differenz von Tatsachen und Meinungen, Wahrheit und Täuschung nicht aufgibt. Auch der von Emma Dowling, Silke van Dyk und Stefanie Graefe formulierte "Einspruch“ stellt die Frage nach den Möglichkeiten einer links-emanzipatorischen Kritik der derzeit vergleichsweise erfolgreichen rechtspopulistischen Politikangebote. Dabei bürsten die drei Autorinnen allerdings 
nicht den „Rechtspopulismus“ selbst gegen den Strich, sondern die in der einschlägigen linken Kritik-Debatte wahrgenommene Tendenz, die Verantwortung für den gegenwärtigen Erfolg der Rechten nicht zuletzt bestimmten emanzipatorischen Theorie- und Politikkonzepten, namentlich der „Identitätspolitik“ bzw. „Gendertheorie“ zuzuschreiben. Sie diagnostizieren hier eine - überaus problematische - Rückkehr von „Hauptwiderspruchs-Logiken“ in der Linken, die ihrerseits auf (zu) einfachen Gegenüberstellungen von „eigentlicher“ Realität und vermeintlichen LuxusProblemen basiert und auf diese Weise hinter bereits erreichte emanzipatorische Standards zurückfällt. Alex Demirović nimmt jüngere politische Bezugnahmen auf "Gerechtigkeit“ als Anlass zu einer kritischen Auseinandersetzung mit Ansätzen der Gerechtigkeitsphilosophie und der empirischen Gerechtigkeitsforschung. Gezeigt wird, dass eine Kritik, die sich auf Ungerechtigkeit beruft, paradoxe Wirkungen hervorbringt und die Akteure in ein spezifisches, nämlich moralisches Herrschaftsverhältnis verwickelt, sie also nicht zu einem kritischen Handeln anregt, sondern ratlos macht. Dies wird mit Rückgriff auf Marx' und Horkheimers Kritik der ideologischen Form der Moral erklärt.

Sozialwissenschaftliche Gesellschaftskritik bezieht sich oftmals auf Karl Marx. Vor 150 Jahren, im September 1867, erschien die erste Auflage von Das Kapital. Auch wenn die Resonanz zunächst verhalten war und sein Freund Friedrich Engels mit ein paar Rezensionen unter falschem Namen nachhelfen musste, so wurde es doch zu einer der einflussreichsten ökonomie- und gesellschaftskritischen Schriften - mit einer Ausstrahlung in nahezu alle wissenschaftlichen Disziplinen. Drei Beiträge in unserem zweiten Schwerpunkt diskutieren ausgewählte Fragen.
Nach 150 Jahren ist Das Kapital noch immer eine Herausforderung. Erst seit wenigen Jahren sind alle Manuskripte, die dem Kapital zugrunde liegen, in der Marx-Engels Gesamtausgabe (MEGA ${ }^{2}$ ) zugänglich. Die Bedingungen für eine textkritische Lektüre sind also heute besser als je zuvor. Gleichzeitig ist man mit einer Vielzahl an Interpretationen konfrontiert, die eine „unschuldige Lektüre“ (Althusser) des Kapital gar nicht möglich machen. Jede Lektüre ist durch Traditionen vorgeprägt, deren Linien kaum mehr sichtbar oder gar bekannt sind.

Michael Heinrich diskutiert in seinem Essay die Bedingungen der paradoxen Rezeption des Kapital, denn aus Marx, dem Kritiker der klassischen politischen Ökonomie, wurde der letzte große Vertreter dieser Schule gemacht. Das wird anhand einiger Beispiele impliziter Kritik aufgezeigt, etwa anhand der Frage, was in der marxschen Theorie von Wert und Kapital wirklich neu war. Darüber hinaus präsentiert er einige stereotype Beispiele einer zeitgenössischen Marx-Kritik und skizziert schließlich den unvollendeten Zustand des Kapital und was wir von den kommenden Publikationen in der immer noch unabgeschlossenen MEGA ${ }^{2}$ erwarten können. Louis Althusser sprach vom ,schrecklichen ersten Abschnitt" des Kapital und legte nahe, diesen bei der Lektüre zu überspringen. Für Marx-Lektüren, die in der Werttheorie und Fetisch-Konzeptionen einen Kern seiner Kritik erkennen, ist Althussers Intervention nicht nachvollziehbar. Lukas Egger bringt beide Interpretationen in einen Dialog, nicht mit dem Ziel, zu zeigen, dass sie miteinander kompatibel sind, sondern herauszustellen, warum der epistemologische Zugriff Althussers nichts mit der marxschen Werttheorie anfangen kann.

Hans-Peter Büttner stellt in seinem Beitrag eine im deutschsprachigen Raum 
kaum bekannte Interpretation des sogenannten Transformationsproblems vor, die sogenannte Temporal Single-System Interpretation (TSSI), die im angelsächsischen Raum seit Jahren recht breit diskutiert wird. Diese Diskussion zum dritten Band des Kapital ist zum einen relevant, weil das sogenannte Transformationsproblem gerne dafür herhalten muss, die marxsche Theorie als solche infrage zu stellen (siehe auch den Beitrag von Michael Heinrich); die Diskussion ist aber auch deshalb von Interesse, weil sie unterschiedliche Lesarten, Darstellungsfragen und Interpretationen zu Begriffen eröffnet, die sonst eher entlang der ersten Kapitel des Kapital geführt werden.

Außerhalb des Schwerpunkts führen wir die Debatte fort, die mit der PROKLA 185 angestoßen wurde und bereits einige Repliken provoziert hatte. Andrea Kretschmann und Aldo Legnaro nehmen den PROKLA-Titel „Ausnahmezustand“ zum Anlass, den - auch von der PROKLARedaktion strapazierten - Begriff mit dem Ziel zu differenzieren, seine unterschiedlichen Dimensionen genauer in den Blick zu bekommen.
Daniel Mullis hingegen kritisiert die Einseitigkeit vieler Zeitdiagnosen, die primär die „negative“ Seite herausstellen. Dabei werde ohne Not verhindert, auch die Möglichkeitsfenster zur Kenntnis zu nehmen, die in einer polarisierten gesellschaftlichen Situation existieren. Das Erstarken der extremen Rechten, mitunter bei Wahlen, ist eine zentrale Dimension pessimistischer Zeitdiagnosen. Vor allem deshalb, weil konservative Kräfte meinen, dem etwas entgegensetzen zu können, indem sie selbst einen autoritären und nationalistischen Kurs verfolgen. Auch deshalb wurden die Wahlen in Frankreich mit großer Spannung verfolgt. Felix Syrovatka geht auf die Präsidentschafts- und Parlamentswahlen ein und klärt, wie diese einzuschätzen sind und was dieses politische Erdbeben für die politische Landschaft in Frankreich bedeutet.

Die PROKLA-Redaktion dankt Emma Dowling, Silke van Dyk, Stefanie Graefe und Tilman Reitz für die Initiative zu einem Schwerpunkt zu Gesellschaftskritik. 


\section{PROKLA 189: Kämpfe um die globale Inwertsetzung von Land und Rohstoffen}

Seit Anfang der 2000er Jahre kommt es zu einer neuen Welle von Einhegungen von Land und Natur. Ursachen hierfür sind die Vielfachkrise des Kapitalismus, die Ausweitung imperialer Lebensweisen im Globalen Norden und Süden und die hiermit verbundene stark gestiegene Nachfrage nach Agrarprodukten, bergbaulichen Rohstoffen (Mineralien, Metalle, seltene Erden, Erze) und fossilen und erneuerbaren Energieträgern (Öl, Gas, Kohle, Biomasse, pflanzliche Rohstoffe). Darüber hinaus sorgen Hedgefonds und andere Finanzmarktinstrumente für eine Erschließung von Natur als neue und sichere Anlagefelder für überakkumuliertes Kapital. Dies geht mit zunehmend negativen ökologischen und sozialen Folgen einher. Weltweit verlieren Menschen ihr Land zu Gunsten kommerzieller großfächiger Agrarproduktion sowie von Bergbau-, Klimaschutz- und Infrastrukturprojekten. Der Heftschwerpunkt diskutiert die aktuellen, zum Teil aggressiven Bemühungen um die Sicherung des Zugriffs auf Rohstoffe und Land, die neue Konflikte und Kämpfe ausgelöst und alte verschärft haben. Aktuelle Inwertsetzungsprozesse von Land und Rohstoffen sind häufig Teil nationaler und internationaler Entwicklungsstrategien. Weltweit leisten Bauern und Bäuerinnen, AnwohnerInnen und AktivistInnen und schließlich auch Staaten jedoch Widerstand gegen die Ausweitung der „extraktiven Grenzen“. Diese Kämpfe sind oft nicht nur lokal verankert, sondern über Regionen, Länder und Kontinente hinweg organisiert.

\section{PROKLA 190: Autoritärer Populismus}

Nicht nur in vielen europäischen Ländern existieren starke autoritär-populistische Parteien, die eine nationalistische Zielsetzung vertreten, mit ihrer AlarmismusRhetorik vielfach für teils protektionistische, teils neoliberale Politiken eintreten und einen Kulturkampf betreiben, in dessen Zentrum die Kritik an der sogenannten Genderideologie und der politischen Korrektheit steht. Einerseits sind diese Parteien durchaus für einen starken Nationalstaat, andererseits verfolgen sie in bestimmten Bereichen das neoliberale Ziel des Abbaus des Staates. Nach über einem Jahr der Erfahrungen mit der Präsidentschaft von Donald Trump und seinen rechten Beratern stellt sich die Frage nach den konkreten Entwicklungen in den USA und den Regierungspraktiken der neuen Administration. Das Heft wird sich mit den autoritär-populistischen Parteien, ihren politischen Zielen, den Wahlen und den politischen Konstellationen in den entsprechenden Ländern befassen. Es widmet sich den politischen Zielen der autoritär-populistischen Parteien, den Wahlen und den politischen Konstellationen. Dies schließt die programmatischen Diskussionen, das Wahlverhalten und die Unterstützung durch verschiedene Teile der Bevölkerung mit ein. 


\section{Der PROKLA Förderverein}

Die PROKLA erscheint seit 1971 und bietet politisch engagierte sozialwissenschaftliche und ökonomische Analysen. Allein von den Verkaufserlösen kann sich die PROKLA nicht finanzieren und in die Abhängigkeit von Parteien oder großen Verlagen wollte sie sich nie begeben. Deshalb wird die PROKLA von einem Förderverein herausgegeben, der „Vereinigung zur Kritik der politischen Ökonomie e.V.", die jährlich in ihrer Vollversammlung die Redaktion der Zeitschrift wählt und die nächsten Themenschwerpunkte diskutiert.

Kritische Sozialwissenschaft kann nicht dem Markt überlassen werden. Ohne solidarische Strukturen und finanzielle Unterstützung sind Zeitschriften wie die PROKLA kaum möglich. Die regelmäßigen finanziellen Beiträge der Vereinsmitglieder ermöglichen das Erscheinen der PROKLA, sie schaffen die Voraussetzungen für Kontinuität und Planbarkeit, wie sie für die Redaktionsarbeit unabdingbar sind. Wir freuen uns über weitere Mitglieder, regelmäßige Spenden oder einmalige Zuwendungen. Weitere Informationen teilen wir gerne perE-Mail mit (redaktion@prokla.de).

Vereinigung zur Kritik der politischen Ökonomie e.V.

IBAN: DE17 100100100538135100 - BIC: PBNKDEFF

Postbank Berlin

\section{Verlag Westfälisches Dampfboot}

\section{Étienne Balibar ist Hannah-Arendt-Preisträger für politisches Denken 2017}

Étienne Balibar hat, wie die Preisjuryvorsitzende Prof. Antonia Grunenberg hervorhebt, „in seinen Büchern und im öffentlichen Auftreten ein intervenierendes Denken in der Tradition Hannah Arendts gepflegt. Seine Beiträge haben sich weit über den akademischen Diskurs hinaus an die republikanische Öffentlichkeit gerichtet."

\section{Étienne Balibar}

Europa: Krise und Ende?

aus dem Französischen übersetzt von Frieder Otto Wolf

2016 - 276 Seiten - 24,90€

ISBN: 978-3-89691-842-0

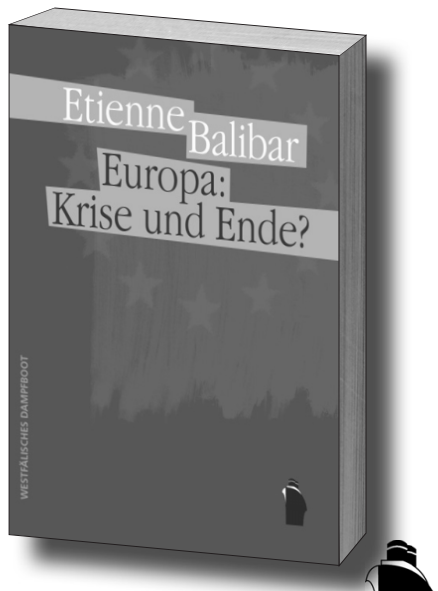

